Archives de sciences sociales des religions

140 | octobre - décembre 2007

Varia

\title{
Evyatar Marienberg, Niddah. Lorsque les juifs conceptualisent la menstruation
}

Paris, Les Belles Lettres, 2003, 366 p.

\section{Sophie Nizard}

\section{(2) OpenEdition}

\section{Journals}

Édition électronique

URL : http://journals.openedition.org/assr/11243

DOI : $10.4000 /$ assr. 11243

ISSN : $1777-5825$

\section{Éditeur}

Éditions de l'EHESS

Édition imprimée

Date de publication : 1 décembre 2007

Pagination : 157-310

ISBN : 978-2-7132-2145-3

ISSN : 0335-5985

\section{Référence électronique}

Sophie Nizard, «Evyatar Marienberg, Niddah. Lorsque les juifs conceptualisent la menstruation », Archives de sciences sociales des religions [En ligne], 140 | octobre - décembre 2007, document 140-59, mis en ligne le 02 juillet 2008, consulté le 21 septembre 2020. URL : http://journals.openedition.org/assr/ 11243 ; DOl : https://doi.org/10.4000/assr.11243

Ce document a été généré automatiquement le 21 septembre 2020

(c) Archives de sciences sociales des religions 


\title{
Evyatar Marienberg, Niddah. Lorsque les juifs conceptualisent la
} menstruation

\author{
Paris, Les Belles Lettres, 2003, 366 p.
}

\section{Sophie Nizard}

1 Cet ouvrage permet d'appréhender la place de la menstruation (croyances, pratiques) dans la culture juive traditionnelle et ses fondements dans les textes. Beaucoup moins étudiés que d'autres pratiques (comme celles de cacherout, de deuil, ou liturgiques) ils sont cependant centraux dans la vie juive traditionnelle.

2 L'originalité et l'intérêt de l'ouvrage, outre sa grande érudition, résident dans sa double approche à la fois historique et comparative. Historique parce qu'au-delà des textes bibliques et talmudiques où les pratiques de purification des femmes après les menstrues sont fortement codifiées, l'auteur tente de saisir l'évolution des croyances et des pratiques de l'Antiquité au Moyen Âge et jusqu'à la période contemporaine. Il montre par exemple comment les menstrues modifient les relations quotidiennes entre un homme et sa femme (relations sexuelles, contacts physiques...). Comparative, parce qu'à chaque fois qu'il le peut, l'auteur met en perspective sources chrétiennes et sources juives concernant cette question, en montrant les influences réciproques des unes sur les autres.

3 Le premier chapitre pose le principe des lois de niddah (le mot niddah a pour racine ndd ou ndh, rejet, expulsion, vagabondage. Utilisé pour désigner la femme qui a ses règles, ou qui à l'issue de ses règles n'a pas procédé aux rites de purification, il qualifie également la "période d'impureté de la femme») selon la halakha (loi religieuse); «Toute femme mariée doit, si elle veut avoir des relations sexuelles avec son conjoint prendre un bain rituel quelques jours après la fin de ses règles. Sinon, elle reste dans un état d'impureté, elle est niddah» (p. 23). L'ablution peut avoir lieu dans la nature (rivière, mer ou source) ou dans une piscine construite à cet effet et répondant à certaines normes: le mikvé. Avant l'ablution elle-même, la femme doit procéder à un nettoyage minutieux de son corps. 
Dans le texte biblique, c'est le chapitre 15 du Lévitique qui fait référence. La souillure des règles, selon ce texte, se transmet par l'intermédiaire des objets avec lesquels la femme est en contact. Un homme qui couche avec une femme ayant ses menstrues contracte cette souillure. Des rites de purification par l'eau et des sacrifices au Temple permettaient de sortir de l'état de niddah. Le chapitre 20 du Lévitique introduit l'idée d'une prohibition des relations sexuelles avec une femme niddah. La sanction encourue par l'homme et la femme est celle de karet (retranchement du milieu du peuple). À l'époque talmudique, certaines restrictions viennent s'ajouter aux lois bibliques sur le principe qu'elles constituent des barrières supplémentaires; ainsi le concept des « sept jours propres » ou «blancs » apparaît : tout écoulement de sang, même en dehors des menstrues, doit être suivi d'une période supplémentaire de sept jours, à l'issue de laquelle le bain rituel est autorisé.

5 Les lois appliquées aujourd'hui par les plus pratiquants ont été édictées au Moyen Âge. Elles concernent les interdits liés aux relations sexuelles, mais également à tout contact, y compris par l'intermédiaire d'un objet, entre une femme qui a ses menstrues et son mari. Ainsi « la coutume Rashi » (1040-1105) interdisait à un homme de donner à sa femme niddah une clé de main à main. L'auteur détaille les soins que la femme doit apporter à son corps avant le bain rituel : laver et peigner sa chevelure, nettoyer et couper ses ongles, se dénuder entièrement afin qu'aucun vêtement, objet ni bijou ne viennent s'interposer entre le corps et l'eau. Lors du bain, une autre personne doit être présente pour attester que le corps a été totalement immergé.

Evyatar Marienberg fait référence à un film réalisé par une cinéaste israélienne en 2002, Tehora/Purity: Breaking the Codes of Silence, où des femmes pratiquant ou ayant pratiqué ces lois, s'expriment pour la première fois à l'écran. Ce film, programmé à la télévision française, montrait comment cette pratique était vécue, parfois avec difficulté, par certaines. Élaborées au cours des siècles, ces lois sont considérées dans le monde orthodoxe comme " un pilier de la halakha » (de la loi). À la fin du XIX siècle en Allemagne, elles ont pris le nom de «lois de pureté familiale » mais l'auteur refuse cette désignation forgée sur le modèle de la Reinheit des Familienslebens, dans un contexte où l'idéologie de la pureté prenait en Allemagne un tour singulier.

7 Dans le deuxième chapitre E. Marienberg examine, à partir de ses sources, les références théologiques concernant la " raison d'être » de la menstruation. La première idée avancée (et combattue par les féministes depuis quelques années) est que les règles seraient une malédiction (curse) attachée à la faute d'Ève. Pourtant, rappelle l'auteur, le texte de la Genèse fait référence aux peines de l'enfantement, non aux menstrues. Même dans les sources médiévales chrétiennes on ne retrouve pas une telle idée. En revanche, dans les premières sources juives, ce sont les douleurs des règles et non les règles elles-mêmes qui sont évoquées comme conséquence de la transgression originelle (Avot de Rabbi Nathan, environ III $^{\mathrm{e}}$ siècle). On trouve chez Isaac bar Avdimi un lien entre les règles et la malédiction d'Ève. Cette idée n'était donc pas inconnue chez les sages du Talmud à la fin du III ${ }^{\mathrm{e}}$ siècle en Babylonie. On trouve également (Talmud de Jérusalem, ve siècle) l'idée selon laquelle le sang versé par Ève (elle donne à Adam le fruit défendu, ce qui entraînera leur mort différée) doit être expié par le sang des menstrues. L'auteur se penche enfin sur une accusation courante au Moyen Âge dans le monde chrétien: les hommes juifs, coupables de déicide, seraient soumis aux menstrues. Ces représentations établissent ainsi un lien entre les secrétions sanguines et le meurtre. Ainsi les pertes de sang, réelles ou imaginaires, trouvent leur explication 
comme punition pour des meurtres mythiques. «Tandis que des chrétiens accusaient les juifs d'être les assassins de Jésus, le deuxième Adam, des juifs reprochaient à leurs propres femmes la mort du premier Adam » (p. 61). Au XvI esiècle, la littérature yiddish à caractère moral, à destination des femmes, émet l'idée qu'elles sont l'incarnation d'Ève ; à ce titre elles sont considérées comme meurtrières.

8 Sous-jacente aux interrogations sur la menstruation, extrêmement inquiétante aux yeux des hommes, c'est la question des relations sexuelles avec les femmes en fonction de leur cycle qui apparaît au chapitre III. Deux textes écrits à Worms sont mobilisés : l'un par un auteur chrétien du $\mathrm{x}^{\mathrm{e}}$ siècle, Buchard de Worms, l'autre par un auteur juif du XII ${ }^{e}$ siècle, Eléazar de Worms. Dans les deux textes, le fait de coucher avec une femme qui a ses règles est une transgression, et le fauteur doit faire pénitence : pain et eau pendant dix jours selon Buchard, quarante jours de flagellation, sans viande, ni vin, ni pain frais selon Eléazar.

9 Pourquoi donc le judaïsme, comme le christianisme, et d'autres cultures, ont interdit les relations sexuelles pendant les périodes menstruelles? Pour l'auteur, rejoignant en partie les thèses des féministes, la peur de ce qui relève des organes sexuels et du sang des femmes, la volonté de se protéger contre les forces ou les impuretés qui les caractérisent, la volonté de domination masculine sur les femmes et la possibilité de choisir entre plusieurs femmes en régime de polygamie, ou enfin la volonté des femmes elles-mêmes de maîtriser leur sexualité, sont des hypothèses possibles. Pourtant, comme l'indique $\mathrm{E}$. Marienberg, les auteurs anciens justifient régulièrement les interdits par leur propre corpus culturel et textuel (p. 80).

10 C'est ainsi vers la Bible et plus particulièrement vers le Lévitique qu'il faut se tourner, pour constater l'interdit et prendre connaissance des sanctions qui lui sont assorties: "tous deux seront retranchés du milieu de leur peuple " (Lev. 20,18). Le terme karet (retranchement) est dans la tradition juive une "punition des mains de Dieu » (mort jamais immédiate ni ordonnée par les tribunaux). L'auteur va aux sources chrétiennes et cite les Évangiles. La rencontre de Jésus et de la femme hémorroïsse est le seul cas de guérison miraculeuse où le contact entre Jésus et le malade se fait par le vêtement (pour le reste Jésus guérit par le toucher et/ou par la parole). Cette histoire va alimenter la vision chrétienne de la menstruation pour montrer comment Jésus rejette la séparation imposée aux femmes réglées, contre les avis de ses contemporains. Pourtant les textes chrétiens anciens (environ $\mathrm{III}^{\mathrm{e}}$ siècle), sont contradictoires sur ces questions : certains prônent paradoxalement le respect de la séparation, d'autres luttent contre les interdits ancestraux. Chez Jérôme, l'interdit des relations entre un homme et sa femme pendant les règles est justifié par des raisons médicales; les enfants qui naîtraient d'une telle union risqueraient d'être malformés. Un siècle et demi plus tard, Césaire d'Arles introduit l'idée d'un interdit des relations sexuelles le jour du seigneur et les autres fêtes (p. 96). Selon lui, les enfants issus de ces relations seraient lépreux, épileptiques ou possédés du démon. Énée de Gaza, contemporain de Césaire, fait un pas supplémentaire : en voyant des enfants malformés, on peut penser que leurs parents les ont conçus durant des temps prohibés et que l'on peut donc les punir.

11 L'auteur, citant Ezéchiel 18 qui promet qu'un «fils ne portera pas la faute de son père ni un père la faute de son fils ", pose la question de la persistance des croyances en la répercussion de la faute des parents sur les enfants. Il s'intéresse de manière comparative aux croyances juives sur ces questions. Il mobilise le quatrième livre 
d'Esdras (fin du $\mathrm{i}^{\mathrm{er}}$ siècle) : «Et les femmes en leurs menstruations enfanteront des monstres ", faisant l'hypothèse que les traductions (en grec au II siècle puis en latin au IV siècle) ont contribué à la diffusion de ces croyances en monde chrétien. Si les discussions talmudiques se font l'écho d'un lien entre les actes des parents avant, pendant et après le coït, et le destin et la santé des enfants, le Talmud n'évoque pas de conséquence négative sur les enfants à naître en cas de rapports sexuels pendant les règles. Un tel lien (enfants lépreux comme conséquence d'un rapport durant une période interdite) est pourtant établi dans la littérature midrashique ancienne (Lévitique Rabbah) que l'auteur cite longuement. Deux types de causes sont ainsi mobilisés pour expliquer les conséquences supposées sur les enfants des relations sexuelles durant les menstrues : les causes théologico-médicales et celles relevant d'une punition divine.

12 L'historien se fait ensuite sociologue en abordant le contemporain et en mentionnant les justifications de l'interdit des rapports sexuels pendant les règles dans le monde juif orthodoxe aujourd'hui. L'interdit aurait des propriétés prophylactiques en diminuant le risque de MST et de cancers de l'utérus d'une part, et entretiendrait dans le couple amour et égalité entre les sexes. Les féministes orthodoxes avancent même l'argument d'un contrôle par les femmes du temps du plaisir par la maîtrise du moment du bain rituel. Dans les milieux néo-orthodoxes, l'équilibre du couple est une justification courante, mais plus surprenant, on constate un renouveau de la pratique des lois de niddah dans les communautés non orthodoxes, dans lesquelles on construit de plus en plus de bains rituels. Ceci correspond à l'apparition de nouveaux rites de passage ou de purification : ablutions après la ménopause, pour marquer un divorce ou après un viol.

13 Le quatrième chapitre est consacré aux représentations liées au "fils de la niddah", enfant imaginaire, à travers l'histoire juive. Cet enfant est-il mamzer? Le mamzer est, dans la loi juive, l'enfant né de relations interdites sanctionnées par la peine de retranchement (karet) ou par la peine de mort énoncée par un tribunal terrestre. Ainsi l'enfant fruit d'un inceste ou d'une union adultérine. Le fils de la femme niddah est en principe mamzer. Pourtant le Talmud ne définit pas l'enfant de niddah comme mamzer, même si des textes plus tardifs et surtout les croyances populaires font l'amalgame. Le statut de Ben ha-Niddah (fils de niddah) n'apparaît pas dans le Talmud, mais dans des textes de la deuxième moitié $\mathrm{du} 1^{\mathrm{er}}$ millénaire, où il est présenté de manière extrêmement négative. Un texte juif datant probablement du $\mathrm{x}^{\mathrm{e}}$ siècle, Toledot Yeshu (La généalogie de Jésus) raconte la vie de Jésus en le définissant comme mamzer (issu d'une relation adultérine) et fils de niddah (conçu lors des menstrues): l'auteur fait l'hypothèse que ce texte, violemment antichrétien, circulait dans un contexte de persécution des juifs, et que ceux-ci lançaient des blasphèmes publiquement, sachant qu'ils allaient mourir en martyrs. Ces représentations insultantes de Marie seraient pour E. Marienberg l'une des causes de l'autodafé du Talmud à Paris en juin 1242. De manière générale, ben ha-Niddah a été utilisé comme insulte par les juifs entre eux.

Que devient ce statut au $\mathrm{xx}^{\mathrm{e}}$ siècle? L'auteur cite les responsas d'un des plus illustres décisionnaires du monde ultra-orthodoxe, Moïse Feinstein (1895-1986). Dans une première responsa (1978), il déclare qu'il faut éviter de se marier avec un fils ou une fille de niddah, même si cette personne a des qualités. Pourtant, dans une responsa plus tardive (1981), il affirme qu'une personne craignant Dieu, ayant des qualités, n'est pas enfant de niddah. Feinstein trouve une justification à cette position. Une femme ne respectant pas les lois de niddah peut s'être purifiée sans intention de le faire, au cours 
d'un bain de mer ou dans une piscine, la providence divine veillant à ce que les périodes soient respectées pour cela!

L'auteur soulève enfin la question de la «stérilité religieuse » des femmes ayant un cycle court et ne pouvant aller au mikvé qu'après l'ovulation. Dans ce cas, certains couples ultra-orthodoxes ont recourt à une insémination artificielle. Mais la question reste posée, quid des fruits de ces techniques ? Quoiqu'il en soit, pour E. Marienberg, le ben ha-niddah a encore un bel avenir puisqu'une majorité d'enfants naît aujourd'hui de femmes ne respectant pas ces lois.

Le chapitre $\mathrm{V}$ présente les croyances et les pratiques concernant les rapports entre la niddah et ce que l'auteur appelle le sacré: si la synagogue semble être un lieu de rencontre du peuple avec Dieu, si la présence divine s'y manifeste, les femmes qui ont leurs menstrues y sont-elles les bienvenues? Pour la plupart des communautés juives à travers l'histoire, la présence des femmes à la synagogue semble aller de soi. Dans le Talmud, si des restrictions sont imposées aux femmes dans la sphère privée, aucune ne lui est imposée dans la sphère publique. Dans la Bible, la femme en couches doit se purifier avant d'entrer en contact avec ce qui est sanctifié (se rendre au Temple notamment), mais rien de tel ne figure dans le Talmud concernant la synagogue. Pourtant, au Moyen Âge, la plupart des auteurs, ceux de l'École de Rashi notamment, considéraient qu'un tel évitement était une «bonne habitude». Même opinion chez Joël Ha Levi au XII et $x^{e}{ }^{e}$ siècles. Au XVI ${ }^{e}$ siècle Moïse Ben Israël Isserles, "Rema ", oppose la loi de base autorisant les femmes réglées à entrer à la synagogue et à toucher des livres sacrés et la coutume qui le leur interdit pendant les jours où elles « voient du sang ». Cependant, les jours de fêtes, elles y sont autorisées «car elles auraient de la peine d'être exclues ".

17 En Italie au XVII ${ }^{e}$ siècle, Shabbetai Be'er semble surpris qu'on lui adresse question à ce propos: selon lui «il serait plus raisonnable d'imposer des restrictions aux hommes impurs qui eux ont plus facilement accès aux rouleaux de la Torah » (p. 223). Il semble que ces pratiques d'évitement étaient plus fréquentes en Europe du Nord et de l'Est qu'en Europe du Sud. On trouve également des discussions sur l'habitude des femmes qui avaient leurs menstrues de ne pas porter les yeux sur les rouleaux de la Torah, en raison, suppose l'auteur, de la croyance dans le pouvoir du regard, comme si regarder équivalait à toucher, voire profaner ou détruire.

18 Si les textes qui évoquent les habitudes qu'ont les femmes de ne pas se rendre à la synagogue les attribuent à la volonté des femmes elles-mêmes, l'auteur propose une autre hypothèse qu'il partage avec l'historienne Judith Baskin : jusqu'à quel point ces pratiques d'isolation ont-elles été imposées par les hommes? Éviter la Torah du regard est comparable, pour E. Marienberg, à la pratique des « huttes menstruelles » que les femmes s'imposent dans d'autres cultures, notamment africaines. L'auteur se risque à une comparaison avec le monde chrétien : se rendre à l'église, consommer l'hostie est-il permis aux femmes pendant leurs menstrues? A priori autorisées, certaines pratiques d'évitement sont attestées, notamment en milieu chrétien orthodoxe. Reprenant les catégorisations qu'opèrent les religions entre nature et culture, l'auteur suggère que pour les fidèles, une telle appréhension serait « naturelle » (p. 242).

19 Si le pouvoir des hommes sur les femmes est attesté dans toutes les cultures traditionnelles (voir Françoise Héritier), j'aimerais ici nuancer cette hypothèse en référence à Foucault pour qui le bio-pouvoir émane de tout lieu. Il faut éviter de se représenter de manière simpliste un pouvoir des dominants (ici les hommes) sur les 
dominés (ici les femmes). Si les femmes s'autocensurent sur ces questions, c'est en partie parce qu'elles reprennent à leur compte les stigmates qui pèsent sur elles, et qui relèvent des peurs et représentations sociales liées au sang, à l'impur et au sacré.

Le chapitre VI analyse les croyances liées à la pratique du mikvé. La première concerne la beauté et les caractères moraux de l'enfant qui naîtra de l'union. Le Talmud rapporte que Rabbi Yohanan, maître palestinien du $\mathrm{III}^{\mathrm{e}}$ siècle, réputé pour sa grande beauté, avait l'habitude de s'asseoir à la porte du bain rituel afin que les femmes en sortant aient des enfants aussi beaux et sages que lui. Selon un texte composé entre le $v^{e}$ et le IV siècle, la Baraïta Niddah, si la femme sortant du mikvé croise une « créature négative », l'enfant à naître risque de lui ressembler : chien, âne, ou rustre sont donc à éviter. Si toutefois elle croisait une telle créature, les textes, y compris plus tardifs, lui enjoignent de retourner se baigner. Ces croyances débouchent sur des rites d'évitement pour aller et revenir du mikvé. Ne pas voir, pour préserver l'enfant à naître, et ne pas être vue, pour éviter le mauvais œil ou les mauvaises pensées, le mikvé est analysé comme une pratique fortement érotisée. Comme le montre l'auteur à partir de sources contemporaines, ces croyances sont encore d'actualité dans le monde juif orthodoxe contemporain.

21 Fort de sa connaissance de ce monde, E. Marienberg se risque bien souvent à des analyses anthropologiques ou sociologiques à partir des textes qu'il étudie ; parfois ses hypothèses sont intéressantes, parfois, il semble incapable de répondre aux questions qu'il pose faute de sources historiques: les textes sont une chose, mais les juifs respectaient-ils ces pratiques au Moyen Âge? Les respectent-ils aujourd'hui ? Si $10 \%$ des juifs se disent aujourd'hui orthodoxes, l'auteur estime à la même proportion ceux qui respectent scrupuleusement ces lois.

Même si ces estimations sont plausibles, il faudrait une véritable enquête socioanthropologique pour prolonger l'analyse et l'élargir aux croyances et pratiques contemporaines. Il s'agirait avant tout de ne pas en rester à une connaissance quantitative, car le rapport au corps et à la sexualité reste socialement tabou, notamment en milieu orthodoxe. Enfin, le rapport à soi et à son corps reste central, et les bricolages symboliques, comme le montre l'auteur à propos des féministes, peuvent conduire à de nouvelles formes de croire et à des réélaborations de pratiques hors des milieux orthodoxes. Il n'en reste pas moins que cet ouvrage constitue une étude historique majeure, en langue française, des textes religieux et des imaginaires sociaux de la menstruation, et qu'il ouvre de nouvelles perspectives au croisement des sciences sociales des religions et du genre. 